\title{
SUM: Spectrum Utilization Maximization in Energy-Constrained Cooperative Cognitive Radio Networks
}

\author{
Yan Long, Hongyan Li, Member, IEEE, Hao Yue, Student Member, IEEE, Miao Pan, Member, IEEE, and \\ Yuguang Fang, Fellow, IEEE
}

\begin{abstract}
Cooperative cognitive radio networks (CCRNs) enable secondary users (SUs) to access primary resource by cooperation with active primary users (PUs). For the cooperationgenerated resource, existing schemes in CCRNs allocate the resource only to the relay SUs. However, this may lead to inefficient spectrum utilization, when the relay SUs have poor channel condition or little traffic load for their own secondary transmissions. In this paper, considering user diversity in secondary networks, we focus on network-level throughput optimization for secondary networks, by allowing all SUs to optimally share the cooperation-generated period. Besides, considering the energy constraint on SUs, we formulate the resource allocation problem from long-term perspective, to reflect the time-varying change of user diversity in channel condition, traffic load and energy amount. We present an online SUM scheme to solve the longterm optimization problem. Although a mixed-integer and nonconvex problem is involved in the SUM scheme, we transform the problem into multiple convex subproblems, and then optimally solve it with low computational complexity. Extensive simulations show that the proposed SUM scheme significantly outperforms the existing schemes.
\end{abstract}

Index Terms-cognitive radio, cooperative communication, resource allocation, energy consumption.

\section{INTRODUCTION}

$\mathbf{I}$ N RECENT years, the boosting growth of wireless services in our daily life has led to a dramatic increase in spectrum demands. However, it is reported that the current fixed spectrum allocation of Federal Communications Commission

Manuscript received January 5, 2014; revised May 8, 2014. This work was partially supported by the NSFC under grant 91338115 and 61231008, National S\&T Major Project under grant 2011ZX03005-004, 2011ZX03004-003 and 2013ZX03004007-003, Shaanxi 13115 Project under grant 2010ZDKG26, National Basic Research Program of China under grant 2009CB320404, Program for Changjiang Scholars and Innovative Research Team in University under grant IRT0852, 111 Project under grant B08038, and State Key Laboratory Foundation under grant ISN1002005 and ISN090305. The work of H. Yue and Y. Fang was partially supported by the U.S. National Science Foundation under grant ECCS-1129062 and CNS-1343356. The work of M. Pan was partially supported by the U.S. National Natural Science Foundation under grants CNS-1343361 and NSF-1137732. The preliminary version has been presented at The 2014 IEEE International Conference on Communications (ICC) [1]

Y. Long and $\mathrm{H}$. Li are with the State Key Laboratory of Integrated Services Networks, Xidian University, Xi'an, China (e-mail: \{ylong@stu, hyli@\}xidian.edu.cn).

H. Yue and Y. Fang are with the Department of Electrical and Computer Engineering, University of Florida, FL, USA (e-mail: \{hyue@, fang@ece.\}ufl.edu).

M. Pan is with the Department of Computer Science, Texas Southern University, TX, USA (e-mail: panm@tsu.edu).

Digital Object Identifier 10.1109/JSAC.2014.141113.
(FCC) fails to utilize spectrum resource efficiently and many licensed spectrum bands are highly under-utilized [2]-[4]. As one of the promising approaches to increase the spectrum utilization, cognitive radio technology enables secondary users (SUs) to dynamically access the licensed spectrum bands from primary users (PUs) [5]-[8].

As a new paradigm in cognitive radio networks, cooperative cognitive radio network (CCRN) integrates cooperative communications with cognitive radio technology [9]. In CCRNs, even if PUs are occupying the licensed spectrum, SUs can still gain transmission opportunities through cooperating with the active PUs. This is different from the traditional cognitive radio networks in [7] where SUs transmit only when PUs are idle, and also different from the transmission scheme in [10], [11] where concurrent transmissions of PUs and SUs happen only if the interference from SUs to PUs is under a certain threshold. To further illustrate CCRNs, we use a toy example in Fig. 1. $P U_{M}$ is transmitting to $P U_{N}$, and in the same geographic area, $S U_{i}$ and $S U_{j}$ both intend to send data to Secondary Access Point (SAP). We assume the direct link $P U_{M}-P U_{N}$ is severely damaged due to the channel fading, and $S U_{i}$ has better channel condition to the primary receiver. In this situation, PUs may choose to cooperate with $S U_{i}$ to increase their transmission rate, shorten their transmission time and save their energy consumption. With this cooperation, the primary transmission is completed earlier than the intended transmission time, and thus, a vacant time period is created. This cooperation-generated period could in return be exploited for secondary transmissions, and in this way, more transmission opportunities are created for the secondary network. For this reason, CCRN brings a win-win situation to both PUs and SUs [12], [13].

Recently, the resource allocation problem in CCRNs has been studied in [13]-[22]. Zhang et al. select relay SUs jointly with a scheduling among PUs and SUs, to maximize the utility of PUs and also provide certain transmission opportunities for SUs [13]. Cao et al. particularly consider the diversity of communication strategies, and maximize the utility of PUs by choosing the optimal communication strategy [14]. Both of them are optimized through Stackelberg game by setting PUs as leaders and SUs as followers in CCRNs. Besides, Khalil et al. extend the static resource allocation problem to dynamic CCRNs and discuss the short-term and long-term rewards of SUs separately [15]. $\mathrm{Xu}$ and $\mathrm{Li}$ focus on an OFDMA based 


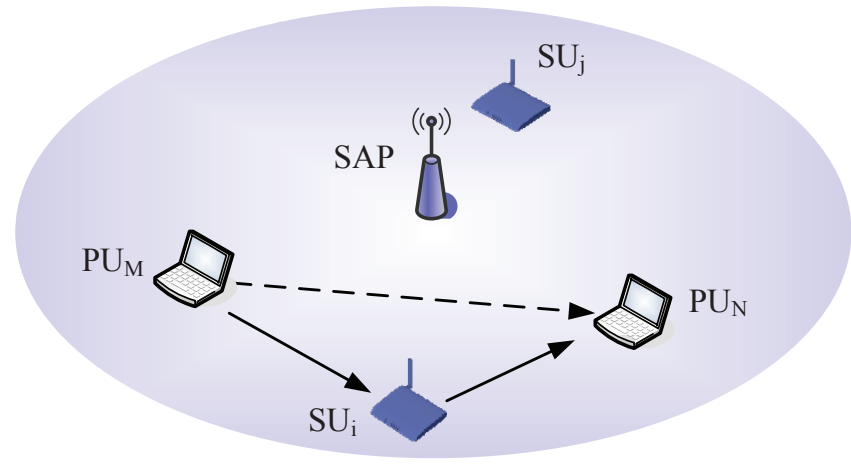

Fig. 1. An illustrative example of CCRNs

multi-channel primary network and take the channel diversity as another optimization domain in CCRNs [16]. Li et al. explore the multi-hop relay selection issue based on a newly proposed FTCO framework [17]. Moreover, SUs with multiple antennas are also introduced in CCRNs, where an SU can perform relay and secondary transmission simultaneously. In this scenario, Hua et al. discuss the resource allocation scheme under a proposed MIMO-CCRN framework [18], and Manna et al. design the power and antenna weights for the secondary transmitters with multiple antennas [19].

An implicit premise behind these existing works is that, the cooperation-generated period is only assigned to the relay SUs, and other SUs cannot transmit in this period. This premise could lead to throughput optimization for relay SUs, but from the view of secondary network operators, maximizing the throughput of whole secondary network may be more important than that of relays. Because of the user diversity in channel condition and traffic load, for the cooperationgenerated period, the best SU for cooperation may not be the best candidate for secondary transmissions. Therefore, allocating the cooperation-generated period among all of SUs may provide more efficient spectrum utilization and higher network-level throughput. For example, in Fig. 1, although $S U_{i}$ relays for PUs, letting $S U_{j}$ transmit in the cooperationgenerated period, instead of $S U_{i}$, would gain more networklevel throughput, especially when $S U_{j}$ maintains better channel condition to SAP than $S U_{i}$, or $S U_{j}$ has heavier traffic load to SAP than $S U_{i}$.

In addition, in an energy-constrained secondary network, the different energy amount of SUs should also be discussed for user diversity. For example, back to Fig. 1, if $S U_{i}$ does not have sufficient energy for its own transmission, selecting $S U_{j}$ to transmit in the cooperation-generated period would be better. Therefor, in energy-constrained networks, user diversity is extended from two domains into three domains: channel condition, traffic load, and available energy. All the three aspects should be jointly considered in the allocation of the cooperation-generated period. Moreover, since energy is directly related to time and is a long-term network parameter, we should optimize the network performance over a long period of time. However, in this long-term optimization, channel condition and traffic arrival is time varying, and also, current resource allocation will directly influence SUs' future traffic load and available energy amount. This makes the user diversity change over time and greatly complicates the resource allocation problem. Hence, how SUs mutually influence with time should be considered in the long-term resource allocation in CCRNs. However, this issue also attracts little attention in the existing works.

In this paper, considering the user diversity of SUs in channel condition, traffic load and energy amount, we propose a novel resource allocation scheme to maximize the long-term network-level throughput in energy-constrained CCRNs. In the long-term optimization, we jointly formulate the resource allocation problem from three aspects: (1) relay selection to choose the relay SUs for active primary transmissions, (2) secondary transmission scheduling to allocate time for secondary transmissions among all SUs, rather than only relays, and (3) power allocation to determine the cooperation power for relay SUs and the transmission power for all SUs for their own secondary transmissions. Note that these three aspects are coupled by the energy constraint since they all determine the energy consumption of SUs. Similar to the secondary service provider (SSP) in [23], to improve the network-level performance for secondary networks, we assume that the SAP is responsible for centralized control and spectrum resource collaboration. We design an online spectrum utilization maximization (SUM) algorithm to solve the long-term network throughput maximization problem. In each control interval, the online SUM scheme involves an admission control problem and a network control problem which jointly optimizes the relay selection, secondary transmission scheduling and power allocation issues. The former admission control problem is linear and easy to solve, while the latter network control problem is a mixed-integer and nonconvex optimization problem, which is hard to solve. But, by exploiting the feature of its formulation, we could optimally solve the network control problem with low complexity. Our major contributions are summarized as follows:

- We optimize the network-level throughput to improve the spectrum utilization for secondary networks. Considering user diversity in terms of channel condition, traffic load and available energy, we allow all SUs to optimally share the cooperation-generated period. This helps SUs utilize spectrum resource in a more flexible and efficient way, and improves network-level throughput performance.

- We focus on long-term network optimization to reflect the energy constraint on SUs and to capture the timevarying feature of practical CCRNs. Based on Lyapunov optimization technique [24], [25], we design an online SUM algorithm to solve the long-term problem without requiring statistical network information. In the online SUM algorithm, the time-varying change of SUs' traffic load and energy amount is reflected through SUs' network layer queue and energy consumption queue, respectively. By running the algorithm over a long period of time, the long-term optimum could be arbitrarily achieved.

- Our online SUM algorithm decouples the long-term optimization problem into an admission control problem and a network control problem in each interval. The admission control problem is linear and easy to solve, while the network control problem is mixed-integer and 
non-convex since it takes relay selection, secondary transmission scheduling and power allocation issues jointly. Although this network control problem is hard to solve, based on the feature of its problem formulation, we transform the network control problem into a series of convex subproblems, and optimally solve it with low computational complexity.

- Simulation results demonstrate that the proposed SUM scheme has great advantages over the previous schemes in terms of long-term secondary network throughput and provides higher spectrum utilization in energyconstrained CCRNs.

The rest of this paper is organized as follows. In Section II, we introduce the system model and parameters in CCRNs. In Section III, we formulate the long-term networklevel throughput optimization problem in energy-constrained CCRNs. Then, we propose an optimal online SUM algorithm to solve the resource allocation problem in Section IV, and present the numerical simulation results in Section V. Finally, we conclude this paper in Section VI.

\section{SySTEM ModeL}

In this section, we present the system model and main system parameters in energy-constrained CCRNs.

We consider a CCRN as shown in Fig. 2. There is a primary network with primary node set $N_{P}$ and link set $L_{P}$. In the same geographic area, there is a secondary network with secondary node set $N_{S}$ and one SAP. Each SU has secondary traffic to send to the SAP. We use capital letters to denote PUs, small letters to denote SUs and 0 to denote SAP. Also, we denote a link by its transmitter-receiver pair. For example, $M N$ represents the primary link from PU $M$ to PU $N, i N$ means the link from SU $i$ to PU $N$, and $i 0$ is the link from SU $i$ to SAP. The above primary network is a general network model. In specific applications, the primary network could be a device-to-device network with separate transceivers on primary links [26], or be an infrastructure-based network with a common transceiver (e.g., a cellular network with base station or a wireless LAN with access point). The secondary network could be a cognitive femtocell network with base station [22], a sensor network with sink node [27], or the architecture proposed in [23] with infrastructure SSP.

Time is discretized into control intervals. In interval $t$, for SU $i$, we use $\lambda_{i}(t)$ to denote the traffic arrival rate at its transport layer, and $A_{i}(t)$ to denote the admitted rate from the transport layer to the network layer. $A_{i}(t)$ is usually bounded by a positive constant $A_{\max }$ to avoid infinite data to the network layer. We assume PUs assess spectrum resource in time-division multiplexing access (TDMA) mode, and in each interval, at most one primary link is activated to avoid interference among PUs. We assume the resource allocation for the primary network has already been done by primary network controller. Given the primary resource allocation results, we have $\eta_{M N}(t)=1$ if the primary link $M N$ is active in interval $t$; otherwise, $\eta_{M N}(t)=0$.

In interval $t$, if primary link $M N$ is active and SU $i$ cooperates as a relay, interval $t$ will be split into a cooperation period and a secondary transmission period (i.e., the

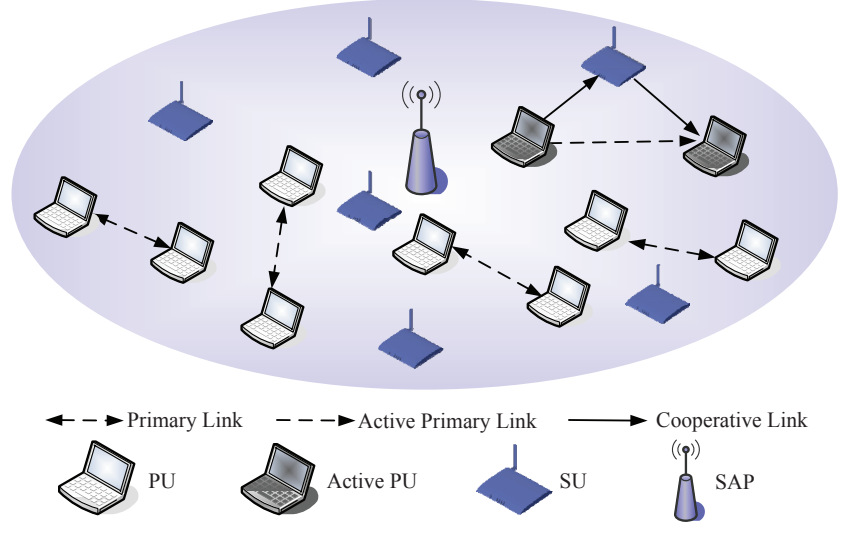

Fig. 2. Network topology of a CCRN

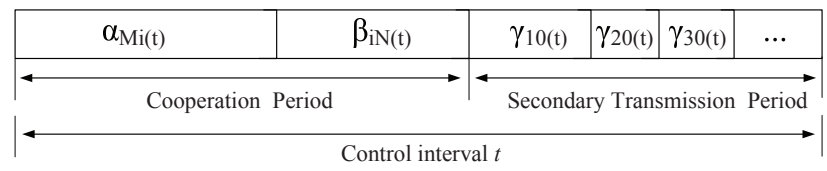

Fig. 3. Time splitting in a control interval

aforementioned cooperation-generated period), as shown in Fig. 3. Similar to the cooperative transmissions in [13], [21], during the cooperation period, $M$ transmits primary traffic to relay $i$ in duration $\alpha_{M i}(t)$, and both $M$ and $i$ cooperatively transmit the primary traffic to $N$ in duration $\beta_{i N}(t)$. Here, this cooperative transmission scheme considers both messages on direct link and relay link at the primary receiver. However, our proposed scheme could also be easily extended to the multi-hop relaying scheme which does not consider the direct transmission at receivers [12], [14], [16]. After the cooperation period, the secondary transmission period is allocated among SUs according to the secondary transmission scheduling strategy, where $\gamma_{i 0}(t)$ represents the time allocated to SU $i$ for its own secondary transmission.

In each interval, we assume that SAP performs the centralized control and resource collaboration in CCRNs, through exchanging information with PUs and SUs over a common control channel. We make this assumption based on the following reasons: First, both PUs and SUs are willing to cooperate because both of them can benefit from cooperation. To facilitate the cooperation, a centralized controller is necessary. Second, since we focus on the optimization of secondary network performance, nodes in primary networks may do not have the interests to optimize such secondary performance. Thus, putting controller in the secondary network side would be more reasonable. Third, by setting SAP as the controller, we move the controlling complexity from primary network side to secondary network side. This minimizes the cooperation overhead to the existing primary networks, and corresponds to the principle of cognitive radio network that PUs should not be influenced too much by secondary networks [9].

We assume channel fading are independent and identically distributed (i.i.d.) across intervals, and independent across different users. Channel gains change over time, but remain constant within each interval, implying channels and nodes in the CCRN are relatively stationary, which is consistent with 
practical applications for wireless LANs. This also hints that the interval will not be too short, for example, the length of the interval could be tens of milliseconds for a slow fading channel [28]. Under such slow fading channel, the time splitting during the interval is meaningful. $h_{M N}(t)$ represents the instantaneous channel gain of link $M N$ in interval $t$. Similarly, we denote $h_{M i}(t), h_{i N}(t)$ and $h_{i 0}(t)$ for links $M i, i N$ and $i 0$, respectively. We assume the transmission power of PUs is fixed and given, which is denoted as $P$. The power of SUs is optimally determined by the power allocation strategy. In interval $t$, for each $\mathrm{SU} i$, the power for cooperation and for secondary transmission are denoted as $P_{i}^{C}(t)$ and $P_{i}^{T}(t)$, respectively. Both $P_{i}^{C}(t)$ and $P_{i}^{T}(t)$ are bounded by the maximum power $P_{\max }$. Without loss of generality, we assume the length of a control interval is 1 , the spectrum bandwidth is 1 , and the noise power is $N_{0}$.

\section{JOINT OPTIMIZATION FORMULATION}

In this section, we mathematically formulate the long-term network-level throughput optimization problem under energyconstrained CCRNs.

\section{A. Relay Selection Constraint}

For simplicity, we assume that in each interval, at most one $\mathrm{SU}$ is selected as the relay for the active primary link, i.e.,

$$
\eta_{M N}(t) \sum_{i \in N_{S}} \theta_{M N}^{i}(t) \leq 1,
$$

where $\theta_{M N}^{i}(t)=1$ means that the $\mathrm{SU} i$ is selected as the relay for primary link $M N$; otherwise, we set $\theta_{M N}^{i}(t)=0$.

\section{B. Cooperation Constraints for SUs and PUs}

For a primary transmission from $M$ to $N$, provided that the Decode-and-Forward mode is adopted at relay $\mathrm{SU} i$, and maximal ratio combining is exploited at the primary receivers [29], [30], then the primary transmission rate with cooperation can be calculated as,

$$
\begin{aligned}
& R_{M N}^{i}(t)=\min \left\{\theta_{M N}^{i}(t) \eta_{M N}(t) \alpha_{M i}(t) \log \left(1+\frac{\left|h_{M i}(t)\right|^{2} P}{N_{0}}\right),\right. \\
& \left.\theta_{M N}^{i}(t) \eta_{M N}(t) \beta_{i N}(t) \log \left(1+\frac{\left|h_{M N}(t)\right|^{2} P}{N_{0}}+\frac{\left|h_{i N}(t)\right|^{2} P_{i}^{C}(t)}{N_{0}}\right)\right\} .
\end{aligned}
$$

Here, this rate equation considers both direct link and relay link at the receiver. But for a multi-hop relaying scenario without direct link, the proposed scheme also works by just removing the constant term $\frac{\left|h_{M N}(t)\right|^{2} P}{N_{0}}$ from $\theta_{M N}^{i}(t) \eta_{M N}(t) \beta_{i N}(t) \log \left(1+\frac{\left|h_{M N}(t)\right|^{2} P}{N_{0}}+\frac{\left|h_{i N}(t)\right|^{2} P_{i}^{C}(t)}{N_{0}}\right)$.

With cooperative communications, all the data received by the relay SU $i$ from PU $M$ in period $\alpha_{M i}(t)$ must be delivered from $i$ to PU $N$ in period $\beta_{i N}(t)$ [16]. Therefore, we have the following cooperation constraint for SUs:

$$
\begin{gathered}
\theta_{M N}^{i}(t) \eta_{M N}(t) \alpha_{M i}(t) \log \left(1+\frac{\left|h_{M i}(t)\right|^{2} P}{N_{0}}\right) \leq \\
\theta_{M N}^{i}(t) \eta_{M N}(t) \beta_{i N}(t) \log \left(1+\frac{\left|h_{M N}(t)\right|^{2} P}{N_{0}}+\frac{\left|h_{i N}(t)\right|^{2} P_{i}^{C}(t)}{N_{0}}\right) .(2)
\end{gathered}
$$

When constraint (2) holds, then $R_{M N}^{i}(t)$ could be rewritten as $R_{M N}^{i}(t)=\theta_{M N}^{i}(t) \eta_{M N}(t) \alpha_{M i}(t) \log \left(1+\frac{\left|h_{M i}(t)\right|^{2} P}{N_{0}}\right)$.
For PUs, the primary transmission rate under cooperation mode should be no less than that under non-cooperation mode, so that PUs have the incentive to cooperate. Hence, we have the cooperation constraint for PUs as below,

$$
\begin{aligned}
& \eta_{M N}(t) \log \left(1+\frac{\left|h_{M N}(t)\right|^{2} P}{N_{0}}\right) \leq \\
& \sum_{i \in N_{S}} \theta_{M N}^{i}(t) \eta_{M N}(t) \alpha_{M i}(t) \log \left(1+\frac{\left|h_{M i}(t)\right|^{2} P}{N_{0}}\right)+ \\
& \quad\left(1-\sum_{i \in N_{S}} \theta_{M N}^{i}(t)\right) \eta_{M N}(t) \log \left(1+\frac{\left|h_{M N}(t)\right|^{2} P}{N_{0}}\right),
\end{aligned}
$$

where the left-hand-side (LHS) represents the primary transmission rate over direct link $M N$. The right-hand-side (RHS) includes two parts: if the primary transmission is cooperated with an SU, i.e., $\sum_{i \in N_{S}} \theta_{M N}^{i}(t)=1$, the RHS equals to $\sum_{i \in N_{S}} R_{M N}^{i}(t)$; while if the primary transmission is under non-cooperation mode, i.e., $1-\sum_{i \in N_{S}} \theta_{M N}^{i}(t)=1$, the RHS equals to LHS, which makes constraint (3) always satisfied.

\section{Secondary Transmission Scheduling Constraint}

Recall that the length of an interval is 1 , so the summation of all durations should satisfy,

$$
\begin{gathered}
\sum_{M N \in L_{P}} \eta_{M N}(t)\left[\sum_{i \in N_{S}} \theta_{M N}^{i}(t)\left(\alpha_{M i}(t)+\beta_{i N}(t)\right)+\right. \\
\left.\left(1-\sum_{i \in N_{S}} \theta_{M N}^{i}(t)\right)\right]+\sum_{i \in N_{S}} \gamma_{i 0}(t) \leq 1 .
\end{gathered}
$$

In constraint (4), when all primary links are inactive, i.e., $\sum_{M N \in L_{P}} \eta_{M N}(t)=0, \sum_{i \in N_{S}} \gamma_{i 0}(t) \leq 1$ holds, meaning that the interval could be totally used for secondary transmissions. However, in the case where a primary link is active and no SU is selected as the relay, constraint (4) becomes $1+\sum_{i \in N_{S}} \gamma_{i 0}(t) \leq 1$, which means SUs cannot access this interval for their own secondary transmissions. Moreover, if a primary link is active and an SU is selected as the relay, we have $\alpha_{M i}(t)+\beta_{i N}(t)+\sum_{i \in N_{S}} \gamma_{i 0}(t) \leq 1$. This implies that the summation of durations $\alpha_{M i}(t), \beta_{i N}(t)$ and $\gamma_{i 0}(t)$ cannot exceed 1 .

\section{Secondary Queue Stability Constraint}

Each SU $i$ maintains a queue $Q_{T_{i}}(t)$ on its transport layer and a queue $Q_{N_{i}}(t)$ on network layer. The corresponding queue updating equations are,

$$
\begin{aligned}
Q_{T_{i}}(t+1)= & {\left[Q_{T_{i}}(t)-A_{i}(t)\right]^{+}+\lambda_{i}(t) } \\
Q_{N_{i}}(t+1)= & {\left[Q_{N_{i}}(t)-\gamma_{i 0}(t) \log \left(1+\frac{\left|h_{i 0}(t)\right|^{2} P_{i}^{T}(t)}{N_{0}}\right)\right]^{+} } \\
& +A_{i}(t),
\end{aligned}
$$

where $[x]^{+}$means $\max \{x, 0\}, \lambda_{i}(t)$ and $A_{i}(t)$ are the traffic arrival rate at transport layer and the admitted rate from the transport layer to network layer, respectively. $\gamma_{i 0}(t) \log (1+$ $\left.\frac{\left|h_{i 0}(t)\right|^{2} P_{i}^{T}(t)}{N_{0}}\right)$ is the instantaneous transmission rate of $\mathrm{SU}$ $i$, calculated based on the secondary transmission scheduling strategy. We assume $\mathbb{E}\left[\left(\gamma_{i 0}(t) \log \left(1+\frac{\left|h_{i 0}(t)\right|^{2} P_{i}^{T}(t)}{N_{0}}\right)\right)^{2}\right]$ is upper bounded by a constant $R_{\text {max }}^{i}$, where the constant $R_{\text {max }}^{i}$ exists for most channel models, e.g., Rayleigh model. 
Our objective is to maximize the secondary network throughput, while keeping the network layer queue $Q_{N_{i}}(t)$ stable on each SU. To this end, for $Q_{N_{i}}(t)$, its long-term averaged input rate (i.e., the admitted rate) must be less than its long-term averaged output rate (i.e., the transmission rate) [24], [31]. That is,

$$
\begin{aligned}
& \lim _{T \rightarrow \infty} \frac{1}{T} \sum_{t=0}^{T-1} \mathbb{E}\left[A_{i}(t)\right]< \\
& \lim _{T \rightarrow \infty} \frac{1}{T} \sum_{t=0}^{T-1} \mathbb{E}\left[\gamma_{i 0}(t) \log \left(1+\frac{\left|h_{i 0}(t)\right|^{2} P_{i}^{T}(t)}{N_{0}}\right)\right] .
\end{aligned}
$$

In addition, note that $A_{i}(t)$ is bounded by $A_{\max }$ and the transport layer queue $Q_{T_{i}}(t)$. We have,

$$
0 \leq A_{i}(t) \leq \min \left\{A_{\max }, Q_{T_{i}}(t)\right\} .
$$

\section{E. Energy Constraint for SUs}

For SU $i$, to avoid exceeding the total energy amount and prolong lifetime, we introduce $E_{\text {ave }}^{i}$ to denote its averaged available energy during an interval. $E_{\text {ave }}^{i}$ is pre-determined and is proportional to the total energy amount of each SU. In fact, in interval $t, \mathrm{SU} i$ 's energy consumption consists of two parts: the cooperation energy $\sum_{M N \in L_{P}} \eta_{M N}(t) \theta_{M N}^{i}(t) \beta_{i N}(t) P_{i}^{C}(t)$ and the secondary transmission energy $\gamma_{i 0}(t) P_{i}^{T}(t)$. We assume $\mathbb{E}\left[\left(\sum_{M N \in L_{P}} \eta_{M N}(t) \theta_{M N}^{i}(t) \beta_{i N}(t) P_{i}^{C}(t)+\gamma_{i 0}(t) P_{i}^{T}(t)\right)^{2}\right]$ is upper bounded by a constant $E_{\max }^{i}$, since all variables $\theta_{M N}^{i}(t), \beta_{i N}(t), \gamma_{i 0}(t), P_{i}^{C}(t)$ and $P_{i}^{T}(t)$ are finite.

We design an energy constraint for each SU to specify that its long-term averaged energy consumption is less than $E_{\text {ave }}^{i}$, which is expressed as,

$$
\begin{aligned}
& \lim _{T \rightarrow \infty} \frac{1}{T} \sum_{t=0}^{T-1} \mathbb{E}\left[\sum_{M N \in L_{P}} \eta_{M N}(t) \theta_{M N}^{i}(t) \beta_{i N}(t) P_{i}^{C}(t)\right. \\
& \left.\quad+\gamma_{i 0}(t) P_{i}^{T}(t)\right]<E_{\text {ave }}^{i} .
\end{aligned}
$$

In order to satisfy constraint (7), we introduce a virtual energy consumption queue $Q_{E_{i}}(t)$ for each SU [32]. The updating equation of the virtual queue is,

$$
\begin{aligned}
& Q_{E_{i}}(t+1)=\left[Q_{E_{i}}(t)-E_{\text {ave }}^{i}\right]^{+}+ \\
& \quad \sum_{M N \in L_{P}} \eta_{M N}(t) \theta_{M N}^{i}(t) \beta_{i N}(t) P_{i}^{C}(t)+\gamma_{i 0}(t) P_{i}^{T}(t) .
\end{aligned}
$$

Similar to the network layer queue $Q_{N_{i}}(t)$, if $Q_{E_{i}}(t)$ is stable, its input rate will be less than its output rate [24]. In that case, the energy constraint (7) holds.

\section{F. Objective Function}

Since we consider the network-level throughput, the objective is to maximize the secondary network throughput, which is defined as the summation of long-term averaged admitted rate over all SUs:

$$
\max \sum_{i \in N_{S}} \lim _{T \rightarrow \infty} \frac{1}{T} \sum_{t=0}^{T-1} \mathbb{E}\left[A_{i}(t)\right] .
$$

\section{G. Overall Optimization Problem}

By imposing the aforementioned constraints, to maximize the long-term secondary network throughput with energy constraint, we jointly formulate the relay selection, secondary transmission scheduling and power allocation problems as follows:

$$
\max \sum_{i \in N_{S}} \lim _{T \rightarrow \infty} \frac{1}{T} \sum_{t=0}^{T-1} \mathbb{E}\left[A_{i}(t)\right]
$$

subject to:

(1) $-(7)$

$0 \leq P_{i}^{C}(t) \leq P_{\max }, \quad \forall i \in N_{S}$ and $\forall t$

$0 \leq P_{i}^{T}(t) \leq P_{\max }, \quad \forall i \in N_{S}$ and $\forall t$

$\theta_{M N}^{i}(t) \in\{0,1\}, \quad \forall i \in N_{S}, \forall M N \in L_{P}$ and $\forall t$

$0 \leq \alpha_{M i}(t) \leq 1, \quad \forall i \in N_{S}, \forall M N \in L_{P}$ and $\forall t$

$0 \leq \beta_{i N}(t) \leq 1, \quad \forall i \in N_{S}, \forall M N \in L_{P}$ and $\forall t$

$0 \leq \gamma_{i 0}(t) \leq 1, \quad \forall i \in N_{S}$ and $\forall t$,

where $A_{i}(t), P_{i}^{C}(t), P_{i}^{T}(t), \theta_{M N}^{i}(t), \alpha_{M i}(t), \beta_{i N}(t)$ and $\gamma_{i 0}(t)$ are decision variables, and $P, P_{\max }, A_{\max }, E_{\text {ave }}^{i}$, $N_{0}, Q_{T_{i}}(t), \eta_{M N}(t), h_{M N}(t), h_{M i}(t), h_{i N}(t)$ and $h_{i 0}(t)$ are constants at each interval.

It is noticeable that both in the objective function and constraints (5) and (7) of problem (8), the long-term performance is considered and the statistical network information is required. However, the statistics is hard to obtain in practical CCRNs, and this poses a significant challenge to the solution process. For this reason, we develop an optimal online algorithm in Section IV, to solve the long-term problem (8) based only upon the instantaneous network information of each control interval. This is different from the scheme in [22], where statistical information is adopted to achieve the longterm optimum.

\section{Optimal Online SUM Algorithm}

In this section, by exploiting the Lyapunov optimization tool and perspective function [33], we propose an online SUM algorithm to solve the long-term optimization problem (8) with low computational complexity.

From Lyapunov optimization tool, to solve the long-term problem (8), the basic idea is to minimize its Lyapunov driftplus-penalty function, which is expressed as,

$$
\min \triangle L(t)-V \sum_{i \in N_{S}} \mathbb{E}\left[A_{i}(t) \mid Q_{N_{i}}(t), Q_{E_{i}}(t)\right],
$$

where $\triangle L(t)=\frac{1}{2} \mathbb{E}\left[\sum_{i \in N_{S}} Q_{N_{i}}^{2}(t+1)+Q_{E_{i}}^{2}(t+1)-\right.$ $\left.Q_{N_{i}}^{2}(t)-Q_{E_{i}}^{2}(t) \mid Q_{N_{i}}(t), Q_{E_{i}}(t)\right]$ is the Lyapunov drift function of problem (8), and $V \geq 0$ is a pre-defined constant to balance the tradeoff between the network throughput and network delay. Specifically, from Lyapunov drift analysis, with the increase of $V$, the objective of the proposed online algorithm can be arbitrarily close to the optimal throughput, however, with the cost of increasing network delay [34].

Furthermore, the minimization of the Lyapunov drift-pluspenalty function can be achieved through an online SUM algorithm, which addresses an instantaneous admission control problem and a network control problem in each control interval. The details of the algorithm is shown as follows. 


\section{A. Admission Control at SUs}

In interval $t$, given the values of $Q_{N_{i}}(t), Q_{T_{i}}(t), A_{\max }$ and $V$, each SU $i$ locally solves the following optimization problem in terms of its admitted rate $A_{i}(t)$.

$$
\min A_{i}(t)\left(Q_{N_{i}}(t)-V\right)
$$

subject to:

$$
0 \leq A_{i}(t) \leq \min \left\{A_{\max }, Q_{T_{i}}(t)\right\}, \quad \forall i \in N_{S}, \forall t .
$$

Obviously, the optimal $A_{i}(t)$ can be easily obtained according to the threshold rule:

$$
A_{i}(t)=\left\{\begin{array}{ll}
\min \left\{A_{\max }, Q_{T_{i}}(t)\right\}, & \text { if } Q_{N_{i}}(t) \leq V \\
0, & \text { otherwise }
\end{array} .\right.
$$

\section{B. Network Control at SAP}

In interval $t$, SAP first collects the current network setting information from PUs and SUs over the common control channel. Then, SAP solves the optimization problem (11) to attain the optimal relay selection, secondary transmission scheduling and power allocation strategies in the current interval.

$$
\begin{aligned}
& \min \sum_{i \in N_{S}} \sum_{M N \in L_{P}} Q_{E_{i}}(t) \eta_{M N}(t) \theta_{M N}^{i}(t) \beta_{i N}(t) P_{i}^{C}(t)+ \\
& \sum_{i \in N_{S}} \gamma_{i 0}(t)\left(Q_{E_{i}}(t) P_{i}^{T}(t)-Q_{N_{i}}(t) \log \left(1+\frac{\left|h_{i 0}(t)\right|^{2} P_{i}^{T}(t)}{N_{0}}\right)\right)
\end{aligned}
$$

subject to:

(1) $-(4)$

$0 \leq P_{i}^{C}(t) \leq P_{\max }$,

$$
\forall i \in N_{S} \text { and } \forall t
$$

$0 \leq P_{i}^{T}(t) \leq P_{\max }$,

$\forall i \in N_{S}$ and $\forall t$

$\theta_{M N}^{i}(t) \in\{0,1\}$,

$\forall i \in N_{S}, \forall M N \in L_{P}$ and $\forall t$

$0 \leq \alpha_{M i}(t) \leq 1$,

$\forall i \in N_{S}, \forall M N \in L_{P}$ and $\forall t$

$0 \leq \beta_{i N}(t) \leq 1$,

$\forall i \in N_{S}, \forall M N \in L_{P}$ and $\forall t$

$0 \leq \gamma_{i 0}(t) \leq 1$,

$\forall i \in N_{S}$ and $\forall t$,

where $P_{i}^{C}(t), P_{i}^{T}(t), \theta_{M N}^{i}(t), \alpha_{M i}(t), \beta_{i N}(t)$ and $\gamma_{i 0}(t)$ are decision variables. It is noteworthy that in order to solve problem (11), there are two obstacles. One is the binary variable $\theta_{M N}^{i}(t)$, which makes problem (11) into a mixed-integer problem. The other one is the non-convex terms, $\theta_{M N}^{i}(t) \beta_{i N}(t) P_{i}^{C}(t), \gamma_{i 0}(t) P_{i}^{T}(t)$, $\gamma_{i 0}(t) \log \left(1+\frac{\left|h_{i 0}(t)\right|^{2} P_{i}^{T}(t)}{N_{0}}\right)$ and $\theta_{M N}^{i}(t) \beta_{i N}(t) \log (1+$ $\left.\frac{\left|h_{M N}(t)\right|^{2} P}{N_{0}}+\frac{\left|h_{i N}(t)\right|^{2} P_{i}^{C}(t)}{N_{0}}\right)$, in problem (11). Optimizing the mixed-integer non-convex optimization problem (11) is not trivial.

To address these obstacles, we first optimize problem (11) in a simple scenario where the power of SUs (i.e., $P_{i}^{C}(t)$ and $\left.P_{i}^{T}(t)\right)$ is fixed and given. After that, we extend to consider a more general case where $P_{i}^{C}(t)$ and $P_{i}^{T}(t)$ are optimized with a power allocation strategy.

1) Fixed Power Case: In this simple case, SUs adopt fixed power for cooperation and their own transmissions, and thus, $P_{i}^{C}(t)$ and $P_{i}^{T}(t)$ are constants. By fixing $P_{i}^{C}(t)$ and $P_{i}^{T}(t)$, with decision variables $\theta_{M N}^{i}(t), \alpha_{M i}(t), \beta_{i N}(t)$ and $\gamma_{i 0}(t)$, problem (11) becomes a mixed-integer bilinear programming
(MIBLP) problem, which is not easy to solve [35]. However, after fixing binary variables $\left\{\theta_{M N}^{i}(t) \mid i \in N_{S}, M N \in L_{P}\right\}$, the MIBLP reduces to a linear program in terms of $\alpha_{M i}(t)$, $\beta_{i N}(t)$ and $\gamma_{i 0}(t)$, which can be solved easily. For this reason, it is expected to solve the MIBLP problem through reducing it into linear optimization problem by fixing $\left\{\theta_{M N}^{i}(t) \mid i \in\right.$ $\left.N_{S}, M N \in L_{P}\right\}$. Since single relay constraint is specified, there are totally $1+\left|N_{S}\right|$ relay selection strategies, including the strategy of "choosing the direct transmission under noncooperation mode" (e.g., $\left\{\theta_{M N}^{i}(t)=0 \mid i \in N_{S}, M N \in L_{P}\right\}$ ), and the strategy of "choosing SU $i \in N_{S}$ as the single relay under cooperation mode" (e.g., $\left\{\theta_{M N}^{i}(t)=1 \mid M N \in L_{P}\right\}$ and $\left.\left\{\theta_{M N}^{i^{\prime}}(t)=0 \mid i^{\prime} \neq i, i^{\prime} \in N_{S}, M N \in L_{P}\right\}\right)$. Hence, to optimize the MIBLP, we first solve a series of linear problems under each relay selection strategy by fixing its associated $\left\{\theta_{M N}^{i}(t) \mid i \in N_{S}, M N \in L_{P}\right\}$, and then, we choose the minimum one from these results as the optimum. We detail the algorithm to optimize problem (11) under the fixed power case in Algorithm 1.

\begin{tabular}{l} 
Algorithm 1 Network Control Optimization under Fixed \\
Power Case \\
\hline 1: SAP fixes the value of $P_{i}^{C}(t)$ and $P_{i}^{T}(t)$ for each SU \\
in (11), according to a given fixed power strategy; \\
2: SAP solves $(11)$ under the non-cooperation mode, by \\
setting $\left\{\theta_{M N}^{i}(t)=0 \mid i \in N_{S}, M N \in L_{P}\right\}$ in $(11)$; \\
3: For each $\mathrm{SU} i, \mathrm{SAP}$ assumes $i$ is the relay node, and \\
solves $(11)$ under the cooperation mode, by setting its cor- \\
responding $\left\{\theta_{M N}^{i}(t)=1 \mid M N \in L_{P}\right\}$ and $\left\{\theta_{M N}^{i^{\prime}}(t)=\right.$ \\
$\left.0 \mid i^{\prime} \neq i, i^{\prime} \in N_{S}, M N \in L_{P}\right\}$ in $(11)$; \\
4: $\mathrm{SAP}$ compares the $1+\left|N_{S}\right|$ objective values in Steps 2 and \\
3, and chooses the minimum one and its corresponding \\
$\theta_{M N}^{i}(t), \alpha_{M i}(t), \beta_{i N}(t)$ and $\gamma_{i 0}(t)$ as the optimal relay \\
selection and secondary transmission scheduling.
\end{tabular}

In the objective function of problem (11), the weight of variable $\gamma_{i 0}(t)$ is $Q_{E_{i}}(t) P_{i}^{T}(t)-Q_{N_{i}}(t) \log \left(1+\frac{\left|h_{i 0}(t)\right|^{2} P_{i}^{T}(t)}{N_{0}}\right)$, where $Q_{E_{i}}(t), Q_{N_{i}}(t)$ and $h_{i 0}(t)$ reflect the user diversity in energy consumption, traffic load and channel condition, respectively. Note that this weight is a constant when power is fixed. Therefore, in Step 3 of Algorithm 1, when an SU is assumed as the relay, the secondary transmission period will be wholly assigned to one SU which has the lowest weight, i.e., the SU has the best balance among energy consumption, traffic load, and channel condition aspects. Hence, in each interval, although the secondary transmission period is allowed to be used by all SUs, actually, it will be assigned to at most one SU. But we particularly point out that, since the user diversity in traffic load, channel condition and available energy changes over time, different SU will be activated in each interval. Thus, from time-averaged view, the secondary transmission period will be shared and allocated among all SUs.

2) Optimized Power Allocation Case: Different from the fixed power case, in this case, $P_{i}^{C}(t)$ and $P_{i}^{T}(t)$ are decision variables. Since power is unknown, even though we can fix $\theta_{M N}^{i}(t)$ first, the associated subproblem is still non-convex. The key issue to solve problem (11) under the optimized power allocation case is how to solve the non-convex subproblem.

To this end, we exploit the perspective function to trans- 
form the non-convex subproblem into an equivalent convex problem. The perspective function of a given function $f(x)$ is defined as $g(x, y)=y f(x / y)$. It is proved that if function $f(x)$ is convex, then its perspective function $g(x, y)$ is also convex [33].

Specifically, we introduce new variables $B_{i}(t)$ and $G_{i}(t)$, which are defined as,

$$
\begin{aligned}
& B_{i}(t)=\beta_{i N}(t) P_{i}^{C}(t), \\
& G_{i}(t)=\gamma_{i 0}(t) P_{i}^{T}(t) .
\end{aligned}
$$

Then, replacing $P_{i}^{C}(t)$ and $P_{i}^{T}(t)$ by $B_{i}(t)$ and $G_{i}(t)$, we have,

$$
\begin{gathered}
\beta_{i N}(t) \log \left(1+\frac{\left|h_{M N}(t)\right|^{2} P}{N_{0}}+\frac{\left|h_{i N}(t)\right|^{2} P_{i}^{C}(t)}{N_{0}}\right)= \\
\beta_{i N}(t) \log \left(1+\frac{\left|h_{M N}(t)\right|^{2} P}{N_{0}}+\frac{\left|h_{i N}(t)\right|^{2} B_{i}(t)}{N_{0} \beta_{i N}(t)}\right), \\
\gamma_{i 0}(t) \log \left(1+\frac{\left|h_{i 0}(t)\right|^{2} P_{i}^{T}(t)}{N_{0}}\right)= \\
\gamma_{i 0}(t) \log \left(1+\frac{\left|h_{i 0}(t)\right|^{2} G_{i}(t)}{N_{0} \gamma_{i 0}(t)}\right) .
\end{gathered}
$$

In this way, the optimization problem (11) can be rewritten as follows:

$$
\begin{aligned}
& \min \sum_{i \in N_{S}} \sum_{M N \in L_{P}} Q_{E_{i}}(t) \eta_{M N}(t) \theta_{M N}^{i}(t) B_{i}(t)+ \\
& \sum_{i \in N_{S}}\left(Q_{E_{i}}(t) G_{i}(t)-Q_{N_{i}}(t) \gamma_{i 0}(t) \log \left(1+\frac{\left|h_{i 0}(t)\right|^{2} G_{i}(t)}{N_{0} \gamma_{i 0}(t)}\right)\right)
\end{aligned}
$$
subject to:

$$
\begin{aligned}
& \eta_{M N}(t) \sum_{i \in N_{S}} \theta_{M N}^{i}(t) \leq 1 \\
& \forall M N \in L_{P} \text { and } \forall t \\
& \theta_{M N}^{i}(t) \eta_{M N}(t) \alpha_{M i}(t) \log \left(1+\frac{\left|h_{M i}(t)\right|^{2} P}{N_{0}}\right) \leq \\
& \theta_{M N}^{i}(t) \eta_{M N}(t) \beta_{i N}(t) \log \left(1+\frac{\left|h_{M N}(t)\right|^{2} P}{N_{0}}+\frac{\left|h_{i N}(t)\right|^{2} B_{i}(t)}{N_{0} \beta_{i N}(t)}\right) \text {, } \\
& \forall i \in N_{S}, \forall M N \in L_{P} \text { and } \forall t \\
& \eta_{M N}(t) \log \left(1+\frac{\left|h_{M N}(t)\right|^{2} P}{N_{0}}\right) \leq \\
& \sum_{i \in N_{S}} \theta_{M N}^{i}(t) \eta_{M N}(t) \alpha_{M i}(t) \log \left(1+\frac{\left|h_{M i}(t)\right|^{2} P}{N_{0}}\right)+ \\
& \left(1-\sum_{i \in N_{S}} \theta_{M N}^{i}(t)\right) \eta_{M N}(t) \log \left(1+\frac{\left|h_{M N}(t)\right|^{2} P}{N_{0}}\right), \\
& \forall M N \in L_{P} \text { and } \forall t \\
& \sum_{M N \in L_{P}} \eta_{M N}(t)\left[\sum_{i \in N_{S}} \theta_{M N}^{i}(t)\left(\alpha_{M i}(t)+\beta_{i N}(t)\right)+\right. \\
& \left.\left(1-\sum_{i \in N_{S}} \theta_{M N}^{i}(t)\right)\right]+\sum_{i \in N_{S}} \gamma_{i 0}(t) \leq 1, \\
& 0 \leq B_{i}(t) \leq P_{\max }, \\
& \forall i \in N_{S} \text { and } \forall t \\
& 0 \leq G_{i}(t) \leq P_{\max }, \\
& \forall i \in N_{S} \text { and } \forall t \\
& \theta_{M N}^{i}(t) \in\{0,1\} \text {, } \\
& \forall i \in N_{S}, \forall M N \in L_{P} \text { and } \forall t \\
& 0 \leq \alpha_{M i}(t) \leq 1 \text {, } \\
& \forall t \\
& \forall i \in N_{S}, \forall M N \in L_{P} \text { and } \forall t
\end{aligned}
$$
$0 \leq \beta_{i N}(t) \leq 1$
$\forall i \in N_{S}, \forall M N \in L_{P}$ and $\forall t$
$0 \leq \gamma_{i 0}(t) \leq 1$,
$\forall i \in N_{S}$ and $\forall t$,

where $B_{i}(t), G_{i}(t), \theta_{M N}^{i}(t), \alpha_{M i}(t), \beta_{i N}(t)$ and $\gamma_{i 0}(t)$ are decision variables.

Since function $f(x)=-\log (c+x)$ is convex, where $c$ is a constant, then $g(x, y)=-y \log (c+x / y)$, as the perspective function of $f(x)$, is also convex. For this reason, in problem (14), $g\left(\frac{\left|h_{i N}(t)\right|^{2} B_{i}(t)}{N_{0}}, \beta_{i N}(t)\right)=-\beta_{i N}(t) \log (1+$ $\left.\frac{\left|h_{M N}(t)\right|^{2} P}{N_{0}}+\frac{\left|h_{i N}(t)\right|^{2} B_{i}(t)}{N_{0} \beta_{i N}(t)}\right)$ and $g\left(\frac{\left|h_{i 0}(t)\right|^{2} G_{i}(t)}{N_{0}}, \gamma_{i 0}(t)\right)=$ $-\gamma_{i 0}(t) \log \left(1+\frac{\left|h_{i 0}(t)\right|^{2} G_{i}(t)}{N_{0} \gamma_{i 0}(t)}\right)$ are convex. Then, when fixing each $\theta_{M N}^{i}(t)$, the associated subproblem of problem (14) is convex and can be solved efficiently. Since problems (11) and (14) are equivalent, problem (11) can be solved through the optimization of problem (14). Once the optimal value $B_{i}(t)$ and $G_{i}(t)$ in problem (14) are derived, the optimal $P_{i}^{C}(t)$ and $P_{i}^{T}(t)$ in problem (11) can be directly calculated from equations (12) and (13), respectively. The details to solve problem (11) is given in Algorithm 2.

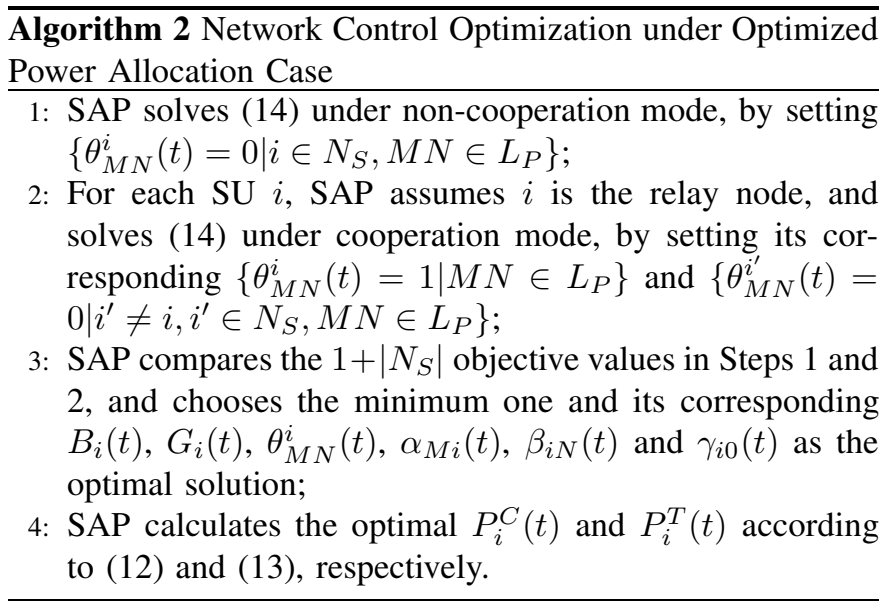

\section{Performance Analysis of Online SUM Algorithm}

In general, at each interval, each SU locally conducts its admission control based on a simple threshold rule (10). For network control under fixed power or optimized power case, SAP solves $1+\left|N_{S}\right|$ linear optimization problems in Algorithm 1 or $1+\left|N_{S}\right|$ convex problems in Algorithm 2, respectively. After that, SAP compares these $1+\left|N_{S}\right|$ results either in Algorithm 1 or Algorithm 2, and chooses the minimum as the optimum. In particular, the linear optimization problem in Algorithm 1 could be easily solved by existing approaches such as simplex method [36], and the convex problem in Algorithm 2 could be efficiently optimized by means like interior-point method [37]. As both simplex method and interior-point method have polynomial-time complexity [37], solving the $1+\left|N_{S}\right|$ linear problems in Algorithm 1 or the $1+\left|N_{S}\right|$ convex problems in Algorithm 2 also has polynomial-time complexity. Besides, comparisons in Algorithm 1 and Algorithm 2 are both performed over a finite set, with the set size proportional to the number of SUs. Hence, the comparisons are also polynomial in the number of SUs. For this reason, both Algorithm 1 and Algorithm 2 can be executed without introducing high computational complexity. 
Overall, based on the analysis above, the admission control problem in Section IV-A and network control problem in Section IV-B can be efficiently solved in each interval. Both two optimization problems only require instantaneous network setting information of each interval, which is much easier to obtain than statistical information required in problem (8). Besides, by exploiting the admission control and network control for a long period of time, the long-term optimum of problem (8) can be arbitrarily closed with the cost of network delay, which is analyzed in Theorem 1.

Theorem 1: For any traffic arrival at transport layers of SUs, the proposed online SUM algorithm could stabilize the secondary network, guarantee the energy constraint of each SU, and optimize the averaged throughput of the secondary network, which are formalized as follows:

$$
\begin{aligned}
& \lim _{T \rightarrow \infty} \frac{1}{T} \sum_{t=0}^{T-1} \sum_{i \in N_{S}} \mathbb{E}\left[Q_{N_{i}}(t)+Q_{E_{i}}(t)\right] \leq \frac{B+V\left|N_{S}\right| A_{\max }}{\epsilon}, \\
& \lim _{T \rightarrow \infty} \frac{1}{T} \sum_{t=0}^{T-1} \sum_{i \in N_{S}} \mathbb{E}\left[A_{i}(t)\right] \geq A^{*}(\epsilon)-\frac{B}{V},
\end{aligned}
$$

where $B=\frac{1}{2} \sum_{i \in N_{S}}\left(R_{\text {max }}^{i}+A_{\text {max }}^{2}+E_{\text {ave }}^{i^{2}}+E_{\text {max }}^{i}\right)$ and $V \geq 0$ are pre-given constants. $A^{*}(\epsilon) \geq 0$ and $\epsilon \geq 0$ are constants given by a stationary randomized algorithm [24], which solves problem (8) based only on statistical network information with control decisions $A_{i}^{S}(t), P_{i}^{C, S}(t), P_{i}^{T, S}(t)$, $\theta_{M N}^{i, S}(t), \alpha_{M i}^{S}(t), \beta_{i N}^{S}(t)$ and $\gamma_{i 0}^{S}(t)$, and ensures the following performance:

$$
\begin{aligned}
& \mathbb{E}\left[\sum_{i \in N_{S}} A_{i}^{S}(t)\right]=A^{*}(\epsilon) \\
& \mathbb{E}\left[A_{i}^{S}(t)\right] \leq \mathbb{E}\left[\gamma_{i 0}^{S}(t) \log \left(1+\frac{\left|h_{i 0}(t)\right|^{2} P_{i}^{T, S}(t)}{N_{0}}\right)\right]-\epsilon \\
& \mathbb{E}\left[\sum_{M N \in L_{P}} \eta_{M N}(t) \theta_{M N}^{i, S}(t) \beta_{i N}^{S}(t) P_{i}^{C, S}(t)+\gamma_{i 0}^{S}(t) P_{i}^{T, S}(t)\right] \\
& \leq E_{\text {ave }}^{i}-\epsilon .
\end{aligned}
$$

Particularly, for $A^{*}(\epsilon)$, we have $\lim _{\epsilon \rightarrow 0} A^{*}(\epsilon)=A^{*}$, where $A^{*}$ is the optimum to problem (8).

From (15), we observe that under the proposed online SUM algorithm, all (virtual) queues in the secondary network could be stabilized with finite queue length. On the other hand, from (16), we can see that by exploiting the proposed online SUM algorithm over relatively long time, the optimum $A^{*}$ could be arbitrarily approached when $V \rightarrow \infty$. However, an increasing $V$ will in return increase the queue length bound in (15), which leads to an increased queue delay.

Proof: See Appendix A.

Besides, to complete cooperation and resource collaboration in CCRNs, the information exchange among users is inevitable. In each interval, each SU solves the admission control problem in a distributed way with its own queue information, and no information exchange is required. To centrally solve the network control problem, at the beginning of each interval, the SAP collects channel condition $h_{M N}(t)$ and $h_{i N}(t)$ from the active primary receiver, channel condition $h_{M i}(t)$ from each $\mathrm{SU}$, and probes the channel condition $h_{i 0}(t)$ by itself. Additionally, the SAP requires queue length information $Q_{N_{i}}(t)$ and $Q_{E_{i}}(t)$ from each SU. After that, the SAP conducts the centralized network control and broadcasts the control decisions to PUs and SUs. All the information exchange is performed on a common control channel. Since the existing schemes in [12], [13], [16] also require channel condition information for decision, compared with them, the difference is that the proposed SUM scheme in addition requires the queue length information of each SU to achieve long-term performance. However, the queue length information could be directly piggybacked in the channel condition message from SU to SAP. As a result, compared with existing algorithms, the proposed SUM algorithm does not occur too much information exchange overhead.

\section{Performance Evaluation}

In this section, we evaluate the performance of the proposed online SUM scheme through extensive simulations.

\section{A. Simulation Setup}

We generate a random primary network with 15 primary links within an area of $1000 \mathrm{~m} \times 1000 \mathrm{~m}$. We run the online SUM scheme over $t_{\max }=4000$ intervals. In each interval, we randomly choose one primary link, and activate it with probability $\operatorname{Pr}_{a}$. The value of $P r_{a}$ is within the range of $[0.4,0.9]$. Larger $P r_{a}$ indicates a busier PU. In the same area, there is a secondary network with randomly located SUs and SAP. The number of SUs increases from 5 to 10 . For each SU, we assume that the traffic arrival at transport layer follows a Poisson process with mean rate $\bar{\lambda}_{i}$, where $\bar{\lambda}_{i}$ is randomly chosen from [0,1Mbps].

We use empirical parameters to model the fading channel [38]. There is a channel centered at $2 \mathrm{GHz}$ with $200 \mathrm{KHz}$ bandwidth. Channel gain is modeled with large-scale and small-scale Rayleigh fading, where large-scale fading is composed by path loss of exponent 3.76 and shadow fading with standard deviation of 10 . For PUs, the transmission power $P$ is fixed to $0.5 W$. For SUs, the power $P_{i}^{C}(t)$ and $P_{i}^{T}(t)$ are optimized through power allocation strategy with the upper bound $P_{\max } \in[0.5 \mathrm{~W}, 2.5 \mathrm{~W}]$. SUs' averaged available energy $E_{\text {ave }}^{i}$ is within the range of $[0,350 \mathrm{~mW}$. We set $N_{0}=7.96 \times 10^{-16} \mathrm{~W}, A_{\max }=10 \mathrm{Mbps}$ and $V=15$.

\section{B. Performance Comparison}

In this topology, we first compare the throughput performance between the SUM-Fixed Power scheme and the proposed SUM scheme. The SUM-Fixed Power scheme, as described in Section IV-B1, considers the SUM formulation with given $P_{i}^{C}(t)$ and $P_{i}^{T}(t)$ under a fixed power strategy. Here, we fix $P_{i}^{C}(t)=P_{i}^{T}(t)=P_{\max }$ in the SUM-Fixed Power scheme. As a counterpart, the SUM scheme, which is studied in Section IV-B2, takes $P_{i}^{C}(t)$ and $P_{i}^{T}(t)$ as decision variables. Numerical results are shown in Fig. 4 and Fig. 5, where $\operatorname{Pr}_{a}=0.8$, the number of SUs is 8 and $\bar{\lambda}_{i}$ is randomly chosen within [0,1Mbps].

In Fig. 4, we plot the throughput performance with respect to the averaged available energy $E_{\text {ave }}^{i} . P_{\max }$ for SUs is set to $2.5 \mathrm{~W}$. It can be observed that the SUM scheme is 


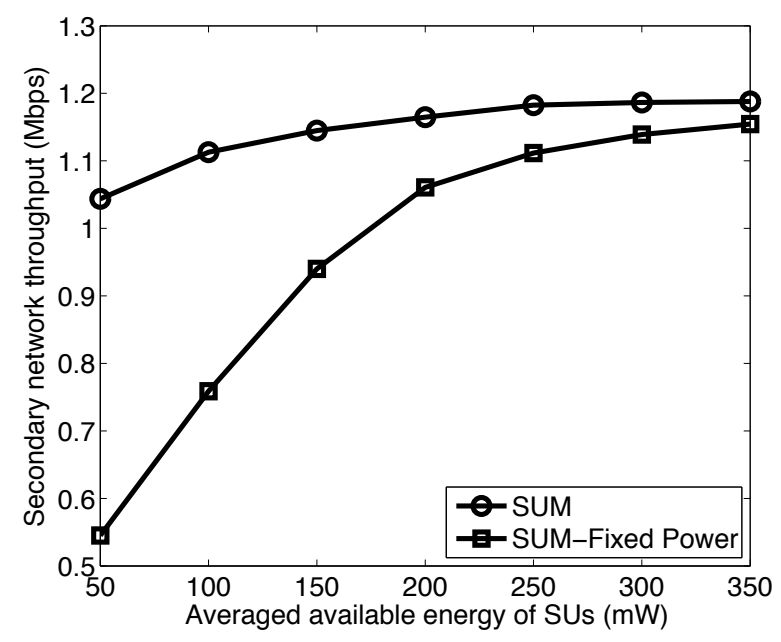

Fig. 4. Secondary network throughput vs. available energy.

always superior to the SUM-Fixed Power scheme in terms of long-term secondary network throughput, while the gap is decreasing with the increase of $E_{a v e}^{i}$. This is because when $E_{\text {ave }}^{i}$ is small, i.e., SUs' available energy is small, to efficiently utilize the constrained energy resource, the role of optimized power allocation in SUM scheme is important. However, when the amount of energy becomes large, the energy resource is sufficient for SUs. Then the advantage of SUM scheme with deliberate power allocation design is not significant.

By fixing $E_{\text {ave }}^{i}=50 \mathrm{~mW}$ and varying the upper bound $P_{\max }$, we demonstrate the impact of $P_{\max }$ on long-term secondary network throughput in Fig. 5. Note that in the SUMFixed Power scheme, the power of SUs is equal to $P_{\max }$. The increase of $P_{\max }$ has two roles for SUs: it can positively lead to an increase of achievable rate, and also negatively result in an increase of energy consumption. Mathematically, the relationship between power and achievable rate is logarithmic, but that between power and energy consumption is linear. Hence, with the increase of $P_{\max }$, the negative effect becomes more dominant than the positive effect. This is why in Fig. 5, the throughput under the SUM-Fixed Power scheme first increases but drops soon.

Then, we compare the length of cooperation period by changing the averaged available energy $E_{\text {ave }}^{i}$ and the maximum power $P_{\max }$. Specifically, running the SUM scheme over $t_{\max }=4000$ intervals, we focus on the intervals when one SU cooperates with one active primary transmission, and calculate the averaged length of cooperation period over these intervals. Results in Table I show that in the SUM scheme, if we increase either $E_{a v e}^{i}$ or $P_{\max }$ for SUs, the averaged length of cooperation period will decrease. The reason is that by having more energy or power for SUs to cooperate, higher primary rate can be achieved. This shortens the cooperative transmission time. Besides, in Table I, we observe that in an interval, the averaged time for cooperation is around 0.6. That is, through cooperation, the averaged time generated for secondary transmission could be around 0.4. This also indicates that the benefit is not trivial when a scheme is particularly designed to efficiently utilize the secondary

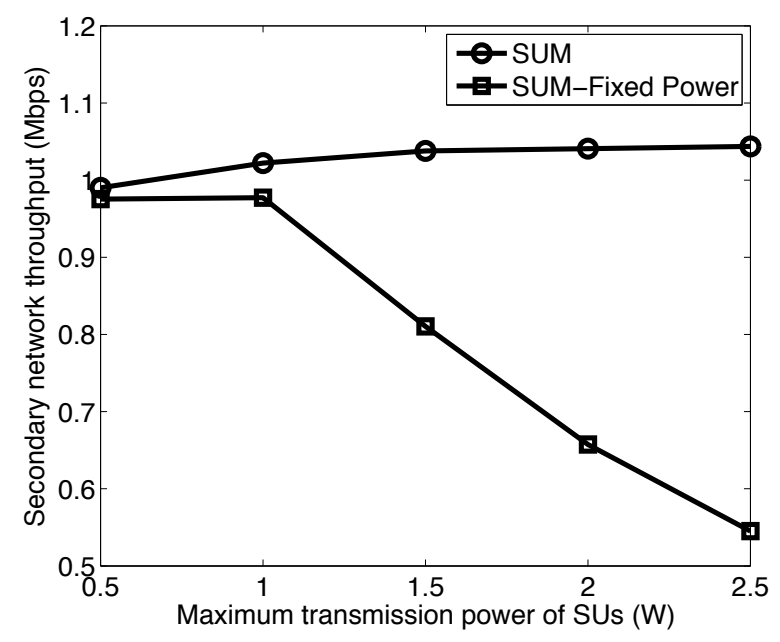

Fig. 5. Secondary network throughput vs. maximum power.

TABLE I

AVERAGED LENGTH OF COOPERATION PERIOD UNDER DIFFERENT AVAILABLE ENERGY AND MAXIMUM POWER.

\begin{tabular}{|c|c|c|c|}
\hline & $P_{\max }=0.5 \mathrm{~W}$ & $P_{\max }=1.5 \mathrm{~W}$ & $P_{\max }=2.5 \mathrm{~W}$ \\
\hline$E_{\text {ave }}^{i}=50 \mathrm{~mW}$ & 0.584 & 0.581 & 0.576 \\
\hline$E_{\text {ave }}^{i}=150 \mathrm{~mW}$ & 0.583 & 0.567 & 0.553 \\
\hline
\end{tabular}

transmission period.

Finally, we compare the throughput performance of the SUM scheme with the conventional simple cooperation scheme (S-Cooperation) [12], [13] and the Non-Cooperation scheme (N-Cooperation) [7]. Here, the S-Cooperation scheme allocates the secondary transmission period only to the relay SUs, and the N-Cooperation scheme does not consider cooperation between primary and secondary networks. Results are shown in Fig. 6 to Fig. 8.

In Fig. 6, $P r_{a}=0.8$, the number of SUs is $8 . P_{i}^{C}(t)$ and $P_{i}^{T}(t)$ are optimized with upper bound $P_{\max }=2.5 \mathrm{~W}$ and $E_{\text {ave }}^{i}$ is within $[0,350 \mathrm{~mW}$. We increase the secondary traffic load by increasing the SUs' Poisson mean rate $\bar{\lambda}_{i}$ from $0.1 \mathrm{Mbps}$ to $0.5 \mathrm{Mbps}$. It can be seen from Fig. 6 that with the increase of $\bar{\lambda}_{i}$, the long-term throughput under three schemes first increases significantly and then keeps steady. The reason is the following: with the increase of $\bar{\lambda}_{i}$, more secondary traffic is available to be admitted, but once the throughput is saturated, the network will fail to admit more traffic.

In Fig. 7, we evaluate the network throughput under three schemes with different number of SUs, by setting $P_{\max }=$ $2.5 \mathrm{~W}, \operatorname{Pr}_{a}=0.8, E_{\text {ave }}^{i} \in[0,350 \mathrm{~mW}]$ and $\bar{\lambda}_{i} \in[0,1 \mathrm{Mbps}]$. The throughput under the SUM scheme and the S-Cooperation scheme increases significantly, since more cooperation opportunities is generated with the increase of SUs. However, under the N-Cooperation scheme, the throughput increases slowly since no cooperation gain is provided. Under the $\mathrm{N}$ Cooperation scheme, the increase of throughput may be from the increase of traffic load as the number of SUs grows.

In Fig. 8, setting the number of SUs as $8, P_{\max }=2.5 \mathrm{~W}$, $E_{\text {ave }}^{i} \in[0,350 \mathrm{~mW}]$ and $\bar{\lambda}_{i} \in[0,1 \mathrm{Mbps}]$, we exam the throughput performance versus PUs' active probability $\mathrm{Pr}_{a}$. When $\operatorname{Pr}_{a}$ grows, i.e., the PUs become busy, the opportunities 


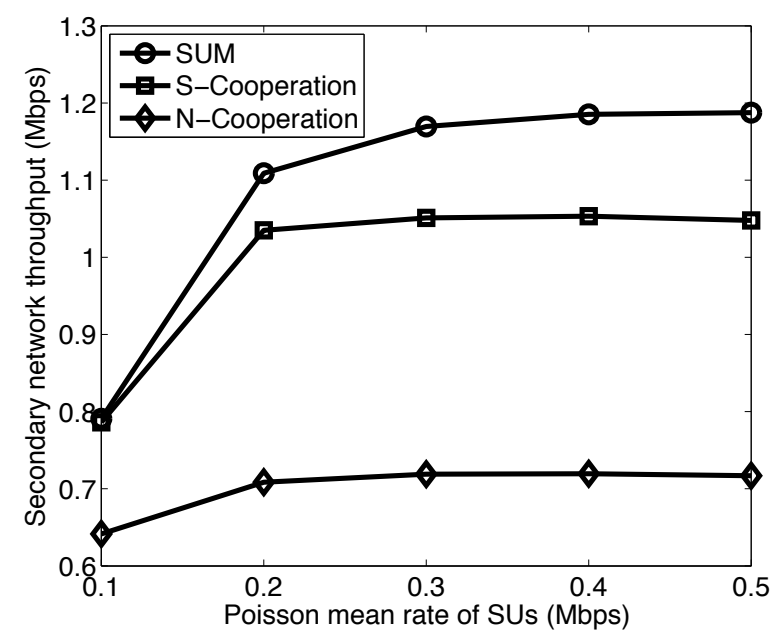

Fig. 6. Secondary network throughput vs. secondary traffic load.

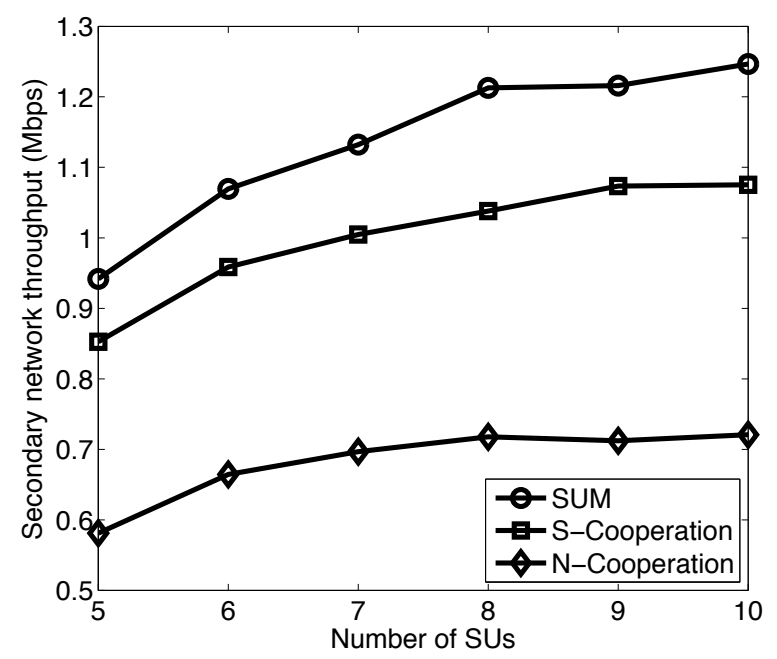

Fig. 7. Secondary network throughput vs. number of SUs.

for secondary transmissions decreases, and the throughput under the three schemes all drops. However, the dropping speed of the SUM scheme is slower than that of the other two schemes, showing that the proposed SUM scheme could create more secondary transmission opportunities than other schemes even when PUs are busy.

In general, it can be seen from Fig. 6 to Fig. 8 that the proposed SUM scheme always outperforms the existing SCooperation scheme and the N-Cooperation scheme, in terms of long-term secondary network throughput. For example, compared with the S-Cooperation scheme, in Fig. 6, the proposed SUM scheme has around 14\% improvement when SUs' Poisson mean rate is 0.5 , in Fig. 7, the SUM scheme provides around $16 \%$ improvement when the number of SUs is 10, and in Fig. 8, the SUM scheme gives around $29 \%$ improvement when PUs' active probability is 0.9. All of these illustrate that, the proposed SUM scheme could utilize the spectrum resource more efficiently and achieve higher network-level throughput for secondary networks.

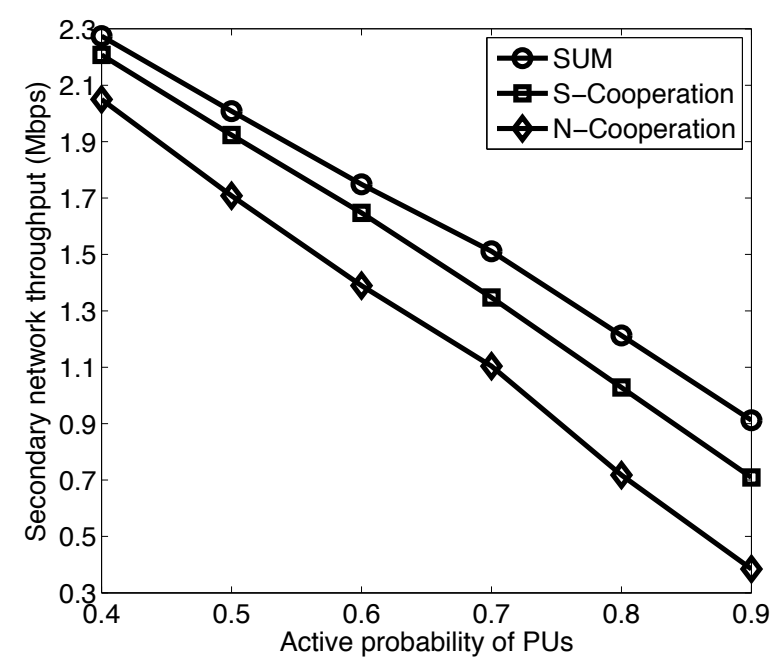

Fig. 8. Secondary network throughput vs. active probability of PUs.

\section{CONCLUSION}

In this paper, we have proposed a novel resource allocation scheme under energy-constrained CCRNs. To improve the spectrum utilization and network-level throughput for secondary networks, we allow all SUs to optimally share the cooperation-generated period, and jointly formulate the relay selection, secondary transmission scheduling and power allocation problems. Considering the energy constraint on SUs, we formulate the resource allocation problem from long-term perspective and design an online SUM scheme to solve the long-term problem with low computationally complexity. Through simulations, we show that compared with existing schemes, the proposed SUM scheme achieves higher secondary network throughput and provides higher spectrum utilization.

\section{APPENDIX A}

\section{PROOF OF THEOREM 1}

To prove Theorem 1, we first define Lyapunov function $L(t)$ as,

$$
L(t)=\frac{1}{2} \sum_{i \in N_{S}}\left(Q_{N_{i}}^{2}(t)+Q_{E_{i}}^{2}(t)\right) .
$$

Based on it, we give the Lyapunov drift $\triangle L(t)$ as,

$$
\triangle L(t)=\mathbb{E}\left[L(t+1)-L(t) \mid Q_{N_{i}}(t), Q_{E_{i}}(t)\right] .
$$

Substituting the queue updating equations $Q_{N_{i}}(t+1)=\left[Q_{N_{i}}(t)-\gamma_{i 0}(t) \log \left(1+\frac{\left|h_{i 0}(t)\right|^{2} P_{i}^{T}(t)}{N_{0}}\right)\right]^{+}+A_{i}(t)$ and $Q_{E_{i}}(t+1)=\left[Q_{E_{i}}(t)-E_{\text {ave }}^{i}\right]^{+}+$ $\left(\sum_{M N \in L_{P}} \eta_{M N}(t) \theta_{M N}^{i}(t) \beta_{i N}(t) P_{i}^{C}(t)+\gamma_{i 0}(t) P_{i}^{T}(t)\right)$ into $\triangle L(t)$, we have,

$$
\begin{aligned}
& \triangle L(t) \leq \frac{1}{2} \sum_{i \in N_{S}} \mathbb{E}\left[\left(Q_{N_{i}}(t)-\gamma_{i 0}(t) \log \left(1+\frac{\left|h_{i 0}(t)\right|^{2} P_{i}^{T}(t)}{N_{0}}\right)\right)^{2}\right. \\
& +A_{i}(t)^{2}+2 Q_{N_{i}}(t) A_{i}(t)+\left(Q_{E_{i}}(t)-E_{\text {ave }}^{i}\right)^{2}+ \\
& \left(\sum_{M N \in L_{P}} \eta_{M N}(t) \theta_{M N}^{i}(t) \beta_{i N}(t) P_{i}^{C}(t)+\gamma_{i 0}(t) P_{i}^{T}(t)\right)^{2}+
\end{aligned}
$$




$$
\begin{aligned}
& 2 Q_{E_{i}}(t)\left(\sum_{M N \in L_{P}} \eta_{M N}(t) \theta_{M N}^{i}(t) \beta_{i N}(t) P_{i}^{C}(t)+\gamma_{i 0}(t) P_{i}^{T}(t)\right) \\
& \left.-Q_{N_{i}}^{2}(t)-Q_{E_{i}}^{2}(t) \mid Q_{N_{i}}(t), Q_{E_{i}}(t)\right] \\
& \leq B+\sum_{i \in N_{S}} \mathbb{E}\left[Q_{N_{i}}(t)\left(A_{i}(t)-\gamma_{i 0}(t) \log \left(1+\frac{\left|h_{i 0}(t)\right|^{2} P_{i}^{T}(t)}{N_{0}}\right)\right)\right. \\
& +Q_{E_{i}}(t)\left(\left(\sum_{M N \in L_{P}} \eta_{M N}(t) \theta_{M N}^{i}(t) \beta_{i N}(t) P_{i}^{C}(t)+\gamma_{i 0}(t) P_{i}^{T}(t)\right)\right. \\
& \left.\left.-E_{\text {ave }}^{i}\right) \mid Q_{N_{i}}(t), Q_{E_{i}}(t)\right],
\end{aligned}
$$

where $B=\frac{1}{2} \sum_{i \in N_{S}}\left(R_{\max }^{i}+A_{\max }^{2}+E_{\text {ave }}^{i^{2}}+E_{\max }^{i}\right)$.

Then, we give the Lyapunov drift-plus-penalty function as follows:

$$
\begin{aligned}
& \triangle L(t)-V \sum_{i \in N_{S}} \mathbb{E}\left[A_{i}(t) \mid Q_{N_{i}}(t), Q_{E_{i}}(t)\right] \\
& \leq B+\sum_{i \in N_{S}} \mathbb{E}\left[A_{i}(t)\left(Q_{N_{i}}(t)-V\right) \mid Q_{N_{i}}(t), Q_{E_{i}}(t)\right]+ \\
& \quad \sum_{i \in N_{S}} \mathbb{E}\left[Q _ { E _ { i } } ( t ) \left(\sum_{M N \in L_{P}} \eta_{M N}(t) \theta_{M N}^{i}(t) \beta_{i N}(t) P_{i}^{C}(t)\right.\right. \\
& \left.+\gamma_{i 0}(t) P_{i}^{T}(t)-E_{\text {ave }}^{i}\right)-Q_{N_{i}}(t) \gamma_{i 0}(t) \log (1+ \\
& \left.\left.\frac{\left|h_{i 0}(t)\right|^{2} P_{i}^{T}(t)}{N_{0}}\right) \mid Q_{N_{i}}(t), Q_{E_{i}}(t)\right] .
\end{aligned}
$$

It can be observed from inequality (20) that, the proposed online SUM algorithm minimizes the second and third parts of the RHS of (20), by exploiting admitted control in Section IV-A and network control in Section IV-B, respectively. As a result, the proposed online SUM algorithm minimizes the RHS of (20) over all feasible algorithms, including the stationary randomized algorithm. Therefore, by substituting control decisions of the stationary randomized algorithm, we have the following result:

$$
\begin{aligned}
& \triangle L(t)-V \sum_{i \in N_{S}} \mathbb{E}\left[A_{i}(t) \mid Q_{N_{i}}(t), Q_{E_{i}}(t)\right] \\
& \leq B+\sum_{i \in N_{S}} Q_{N_{i}}(t) \mathbb{E}\left[A_{i}^{S}(t)-\right. \\
& \left.\gamma_{i 0}^{S}(t) \log \left(1+\frac{\left|h_{i 0}(t)\right|^{2} P_{i}^{T, S}(t)}{N_{0}}\right)\right]+\sum_{i \in N_{S}} Q_{E_{i}}(t) \times \\
& \mathbb{E}\left[\sum_{M N \in L_{P}} \eta_{M N}(t) \theta_{M N}^{i, S}(t) \beta_{i N}^{S}(t) P_{i}^{C, S}(t)+\right. \\
& \left.\gamma_{i 0}^{S}(t) P_{i}^{T, S}(t)-E_{\text {ave }}^{i}\right]-V \mathbb{E}\left[\sum_{i \in N_{S}} A_{i}^{S}(t)\right] \\
& \leq B+\sum_{i \in N_{S}} Q_{N_{i}}(t)(-\epsilon)+\sum_{i \in N_{S}} Q_{E_{i}}(t)(-\epsilon)-V A^{*}(\epsilon),
\end{aligned}
$$

where the last inequality is derived based on (17)-(19).

Taking conditional expectation regarding $Q_{N_{i}}(t)$ and $Q_{E_{i}}(t)$ on both sides of (21) yields,

$$
\begin{aligned}
& \mathbb{E}[L(t+1)-L(t)]-V \mathbb{E}\left[\sum_{i \in N_{S}} A_{i}(t)\right] \leq B+ \\
& \sum_{i \in N_{S}} \mathbb{E}\left[Q_{N_{i}}(t)\right](-\epsilon)+\sum_{i \in N_{S}} \mathbb{E}\left[Q_{E_{i}}(t)\right](-\epsilon)-V A^{*}(\epsilon) .
\end{aligned}
$$

Using telescope sum over (22) from 0 to $t$ leads to,

$$
\begin{aligned}
& \mathbb{E}[L(t+1)]-\mathbb{E}[L(0)]-\sum_{\tau=0}^{t} V \mathbb{E}\left[\sum_{i \in N_{S}} A_{i}(\tau)\right] \leq \\
& (t+1) B-\sum_{\tau=0}^{t} \sum_{i \in N_{S}} \mathbb{E}\left[Q_{N_{i}}(\tau)\right] \epsilon-\sum_{\tau=0}^{t} \sum_{i \in N_{S}} \mathbb{E}\left[Q_{E_{i}}(\tau)\right] \epsilon- \\
& (t+1) V A^{*}(\epsilon) .
\end{aligned}
$$

Since $\mathbb{E}[L(0)]$ is finite, dividing $t+1$ on both side of (23) and taking $t \rightarrow \infty$ brings,

$$
\begin{aligned}
& \lim _{t \rightarrow \infty} \frac{1}{t+1} \sum_{\tau=0}^{t} \sum_{i \in N_{S}} \mathbb{E}\left[Q_{N_{i}}(\tau)+Q_{E_{i}}(\tau)\right] \\
& \leq \frac{B+V\left(\lim _{t \rightarrow \infty} \frac{\sum_{\tau=0}^{t} \sum_{i \in N_{S}} \mathbb{E}\left[A_{i}(\tau)\right]}{t+1}-A^{*}(\epsilon)\right)}{\epsilon} \\
& \leq \frac{B+V\left|N_{S}\right| A_{\max }}{\epsilon}, \\
& \lim _{t \rightarrow \infty} \frac{1}{t+1} \sum_{\tau=0}^{t} \sum_{i \in N_{S}} \mathbb{E}\left[A_{i}(\tau)\right] \geq A^{*}(\epsilon)-\frac{B}{V},
\end{aligned}
$$

where $\lim _{t \rightarrow \infty} \frac{\sum_{\tau=0}^{t} \sum_{i \in N_{S}} \mathbb{E}\left[A_{i}(\tau)\right]}{t+1}$ and $A^{*}(\epsilon)$ are the objective functions under the proposed online SUM algorithm and stationary randomized algorithm, respectively. Since $A_{i}(t) \leq$ $A_{\text {max }}, \forall t$, we have $\lim _{t \rightarrow \infty} \frac{\sum_{\tau=0}^{t} \sum_{i \in N_{S}} \mathbb{E}\left[A_{i}(\tau)\right]}{t+1}-A^{*}(\epsilon) \leq$ $\lim _{t \rightarrow \infty} \frac{\sum_{\tau=0}^{t} \sum_{i \in N_{S}} \mathbb{E}\left[A_{i}(\tau)\right]}{t+1} \leq\left|N_{S}\right| A_{\max }$.

As a result, the performance bounds in (15) and (16) are derived.

\section{REFERENCES}

[1] Y. Long, H. Li, H. Yue, M. Pan, and Y. Fang, "Spectrum utilization maximization in energy limited cooperative cognitive radio networks," in Proc. IEEE ICC, Sydney, Australia, June 2014.

[2] I. F. Akyildiz, W.-Y. Lee, M. C. Vuran, and S. Mohanty, "Next generation/dynamic spectrum access/cognitive radio wireless networks: A survey," Computer Networks, vol. 50, no. 13, pp. 2127 - 2159, September 2006.

[3] Q. Zhao and B. Sadler, "A survey of dynamic spectrum access," IEEE Signal Processing Mag., vol. 24, no. 3, pp. 79-89, May 2007.

[4] Y.-C. Liang, K.-C. Chen, G. Li, and P. Mahonen, "Cognitive radio networking and communications: An overview," IEEE Trans. Veh. Technol., vol. 60, no. 7, pp. 3386-3407, September 2011.

[5] Y. Hou, Y. Shi, and H. Sherali, "Spectrum sharing for multi-hop networking with cognitive radios," IEEE J. Sel. Areas Commun., vol. 26, no. 1, pp. 146-155, January 2008.

[6] C. Gao, Y. Shi, Y. Hou, H. Sherali, and H. Zhou, "Multicast communications in multi-hop cognitive radio networks," IEEE J. Sel. Areas Commun., vol. 29, no. 4, pp. 784-793, April 2011.

[7] M. Pan, P. Li, and Y. Fang, "Cooperative communication aware link scheduling for cognitive vehicular ad-hoc networks," IEEE J. Sel. Areas Commun., vol. 30, no. 4, pp. 760-768, May 2012.

[8] H. Yue, M. Pan, Y. Fang, and S. Glisic, "Spectrum and energy efficient relay station placement in cognitive radio networks," IEEE J. Sel. Areas Commun., vol. 31, no. 5, pp. 883-893, May 2013.

[9] A. Goldsmith, S. Jafar, I. Maric, and S. Srinivasa, "Breaking spectrum gridlock with cognitive radios: An information theoretic perspective," Proc. IEEE, vol. 97, no. 5, pp. 894-914, May 2009.

[10] R. Zhang, Y.-C. Liang, and S. Cui, "Dynamic resource allocation in cognitive radio networks," IEEE Signal Processing Mag., vol. 27, no. 3, pp. 102-114, May 2010.

[11] Y. Xing, C. N. Mathur, M. Haleem, R. Chandramouli, and K. Subbalakshmi, "Dynamic spectrum access with qos and interference temperature constraints," IEEE Trans. Mobile Computing, vol. 6, no. 4, pp. 423-433, April 2007. 
[12] O. Simeone, I. Stanojev, S. Savazzi, Y. Bar-Ness, U. Spagnolini, and R. Pickholtz, "Spectrum leasing to cooperating secondary ad hoc networks," IEEE J. Sel. Areas Commun., vol. 26, no. 1, pp. 203-213, January 2008.

[13] J. Zhang and Q. Zhang, "Stackelberg game for utility-based cooperative cognitiveradio networks," in Proc. ACM MobiHoc, New Orleans, LA, May 2009

[14] B. Cao, J. Mark, Q. Zhang, R. Lu, X. Lin, and X. Shen, "On optimal communication strategies for cooperative cognitive radio networking," in Proc. IEEE INFOCOM, Turin, Italy, April 2013.

[15] K. Khalil, M. Karaca, O. Ercetin, and E. Ekici, "Optimal scheduling in cooperate-to-join cognitive radio networks," in Proc. IEEE INFOCOM, Shanghai, China, April 2011.

[16] H. Xu and B. Li, "Resource allocation with flexible channel cooperation in cognitive radio networks," IEEE Trans. Mobile Computing, vol. 12, no. 5, pp. 957-970, May 2013.

[17] W. Li, X. Cheng, T. Jing, and X. Xing, "Cooperative multi-hop relaying via network formation games in cognitive radio networks," in Proc. IEEE INFOCOM, Turin, Italy, April 2013.

[18] S. Hua, H. Liu, M. Wu, and S. Panwar, "Exploiting mimo antennas in cooperative cognitive radio networks," in Proc. IEEE INFOCOM, Shanghai, China, April 2011.

[19] R. Manna, R. H. Y. Louie, Y. Li, and B. Vucetic, "Cooperative spectrum sharing in cognitive radio networks with multiple antennas," IEEE Trans. Signal Process., vol. 59, no. 11, pp. 5509-5522, November 2011.

[20] T. Jing, S. Zhu, H. Li, X. Cheng, and Y. Huo, "Cooperative relay selection in cognitive radio networks," in Proc. IEEE INFOCOM, Turin, Italy, April 2013.

[21] Y. Yi, J. Zhang, Q. Zhang, and T. Jiang, "Spectrum leasing to multiple cooperating secondary cellular networks," in Proc. IEEE ICC, Houston, TX, December 2011.

[22] R. Urgaonkar and M. Neely, "Opportunistic cooperation in cognitive femtocell networks," IEEE J. Sel. Areas Commun., vol. 30, no. 3, pp. 607-616, April 2012.

[23] M. Pan, P. Li, Y. Song, Y. Fang, and P. Lin, "Spectrum clouds: A session based spectrum trading system for multi-hop cognitive radio networks," in Proc. IEEE INFOCOM, Orlando, FL, March 2012.

[24] M. J. Neely, Stochastic Network Optimization with Application to Communication and Queueing Systems. Morgan and Claypool Publishers, 2010.

[25] H. Ju, B. Liang, J. Li, and X. Yang, "Dynamic joint resource optimization for lte-advanced relay networks," IEEE Trans. Wireless Commun., vol. 12, no. 11, pp. 5668-5678, November 2013.

[26] K. Doppler, M. Rinne, C. Wijting, C. Ribeiro, and K. Hugl, "Device-todevice communication as an underlay to lte-advanced networks," IEEE Commu. Mag., vol. 47, no. 12, pp. 42-49, December 2009.

[27] Y. Zhang, N. Meratnia, and P. Havinga, "Outlier detection techniques for wireless sensor networks: A survey,” IEEE Commun. Surveys Tutorials, vol. 12, no. 2, pp. 159-170, Quarter 2010.

[28] M. Vutukuru, H. Balakrishnan, and K. Jamieson, "Cross-layer wireless bit rate adaptation," in Proc. ACM SIGCOMM, New York, NY, August 2009.

[29] J. Laneman, D. Tse, and G. W. Wornell, "Cooperative diversity in wireless networks: Efficient protocols and outage behavior," IEEE Trans. Inf. Theory, vol. 50, no. 12, pp. 3062-3080, December 2004.

[30] D. Yang, X. Fang, and G. Xue, "Hera: An optimal relay assignment scheme for cooperative networks," IEEE J. Sel. Areas Commun., vol. 30, no. 2, pp. 245-253, February 2012.

[31] H. Ju, B. Liang, J. Li, and X. Yang, "Dynamic power allocation for throughput utility maximization in interference-limited networks," IEEE Wireless Commun. Lett., vol. 2, no. 1, pp. 22-25, February 2013.

[32] M. Neely, "Energy optimal control for time-varying wireless networks," IEEE Trans. Inf. Theory, vol. 52, no. 7, pp. 2915-2934, July 2006.

[33] B. Dacorogna and P. Maréchal, "The role of perspective functions in convexity, polyconvexity, rank-one convexity and separate convexity." Journal of convex analysis, vol. 15, no. 2, pp. 271-284, 2008.

[34] M. Lotfinezhad, B. Liang, and E. Sousa, "Optimal control of constrained cognitive radio networks with dynamic population size," in Proc. IEEE INFOCOM, San Diego, CA, March 2010.

[35] W. Adams and H. Sherali, "Mixed-integer bilinear programming problems," Mathematical Programming, vol. 59, no. 1-3, pp. 279-305, March 1993.

[36] H. Karloff, Linear Programming. Birkhäuser Boston, 1991.

[37] S. Boyd and L. Vandenberghe, Convex Optimization. Cambridge University Press, 2004.

[38] "3GPP TR 36.931 version 11.0.0 release 11, evolved universal terrestrial radio access (E-UTRA); radio frequency $(\mathrm{RF})$ requirements for LTE pico node B," May 2011. [Online]. Available: http://www.etsi.org/deliver/ etsi_tr/136900_136999/136931/09.00.00_60/tr_136931v090000p.pdf

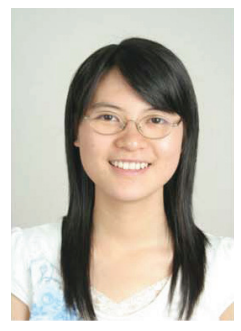

layer optimization.
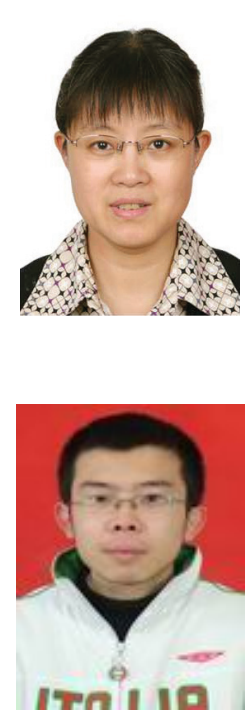

Hao Yue (S'11) received his $\mathrm{BSc}$ degree in Telecommunication Engineering from Xidian University, China, in 2009. He has been working towards the Ph.D. degree in the Department of Electrical and Computer Engineering at University of Florida, Gainesville since August 2009. His research interests include wireless networks and mobile computing, cyber physical systems and security and privacy in distributed systems.

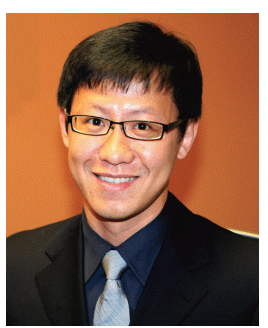

Miao Pan (S'07-M'12) received his BSc degree in Electrical Engineering from Dalian University of Technology, China, in 2004, MASc degree in electrical and computer engineering from Beijing University of Posts and Telecommunications, China, in 2007 and Ph.D. degree in Electrical and Computer Engineering from the University of Florida in 2012, respectively. He is now an Assistant Professor in the Department of Computer Science at Texas Southern University. His research interests include cognitive radio networking and communications, cyber-physical systems, and cybersecurity.

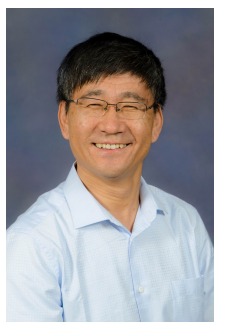

Yuguang "Michael" Fang (S'92-M'97-SM'99F'08) received a BS/MS degree from Qufu Normal University, Shandong, China in 1987, a Ph.D. degree from Case Western Reserve University in 1994 and a Ph.D. degree from Boston University in 1997. He joined the Department of Electrical and Computer Engineering at University of Florida in 2000 and has been a full professor since 2005. He holds a Changjiang Scholar Chair Professorship with Xidian University, China, from 2008 to 2011 . He is a fellow of the IEEE. 